\title{
Acute Lesion of the Intrafusal Muscle of Muscle Spindles: Ultrastructural and Electrophysiological Consequences
}

\author{
Donald C. Quick \\ Department of Anatomy, University of Minnesota, Minneapolis, Minnesota 55455
}

Experiments were performed to functionally separate the neural and muscular elements in muscle spindles. Cat muscle spindles were dissected free in vitro and were transected on both sides of the sensory nerve endings. This resulted in an acute lesion of the intrafusal muscle fibers but spared the sensory endings. Within $\mathbf{4 5} \mathrm{min}$, the intrafusal muscle fibers degenerated to the condition in which there were no myofilaments left in the sensory zone and the muscle fiber membrane had begun to disintegrate. During this degeneration period, and afterward, the sensory nerve fibers continued to fire action potentials. However, the postlesion discharge was usually more irregular than normal, and the mean rate of firing was often dramatically increased. In addition, multiplex frequencies of firing were often observed; that is, the interspike intervals were clustered around 2 or more modal values. Computer modeling of 2 action potential initiators competing for access to the same output line suggests that the multiplexing results from at least one of the competitors having a fraction of its spikes aborted. Overall, the results indicate that the nerve endings have an intrinsic capability to function as sensory receptors in the absence of a muscle substrate.

The sensory function of muscle spindles depends to a large extent on the relations between sensory nerve endings and intrafusal muscle fibers. It is well known that stretching of the intrafusal muscle causes an increase of firing rate in the sensory nerves, and details of the sensory discharge depend on active and passive mechanical properties of the muscle (Boyd, 1976; Poppele and Quick, 1985; Poppele et al., 1979). It is important to determine the nature of interdependence between neural and muscular elements in muscle spindles in order to understand the mechanisms by which mechanical stimuli are transduced to electrophysiological signals. Prior to the work reported here, it was not known whether the nerve fibers were capable of autonomous sensory discharge, exclusive of the presence of a muscle substrate. If it could be determined that the sensory endings were unable to fire on their own, then it would follow that some element of the intrafusal muscle fiber is an essential component of the transducer system. On the other hand, if the sensory units could fire autonomously, then the intrafusal muscle might be considered to be a purely mechanical substrate, acting only as a stimulus modulator. In order to explore further the innate properties of sensory nerve endings in muscle spindles, a model system was tested, involving acute lesion of the intrafusal muscle fibers.

\section{Received Nov. 15, 1985; revised Jan. 13, 1986; accepted Jan. 13, 1986}

This work was supported by National Science Foundation Grant BNS 83-00313.

Correspondence should be addressed to Donald C. Quick, Ph.D., Department of Anatomy, 4-135 Jackson Hall, University of Minnesota, Minneapolis, MN 55455 .

Copyright (C) 1986 Society for Neuroscience $0270-6474 / 86 / 072097-09 \$ 02.00 / 0$

\section{Materials and Methods}

The source of muscle spindles for this work was the tenuissimus muscle of adult cats weighing $3 \mathrm{~kg}$ or more. The cats were anesthetized with pentobarbital (Nembutal, Abbot Laboratories, 35-40 mg/kg) for removal of the muscle and were then immediately killed with an intravenous overdose of anesthetic.

Fresh tenuissimus muscles were immersed in an oxygenated Krebs solution supplemented with amino acids (Poppele et al., 1979), and muscle spindles were located and exposed by dissection. For each dissected spindle, about $1 \mathrm{~cm}$ of the intramuscular nerve (including sensory fibers to the spindle) was dissected free and lifted onto a wire electrode. Electrical activity in the spindle nerve was then amplified and recorded on tape in order to document that the spindlc was active in situ. Next, the spindle was transected on both sides of the equator, leaving only a 1-2 mm segment attached to the nerve. This procedure resulted in an acute lesion of all the intrafusal muscle fibers, but the primary sensory nerve ending was spared. Occasionally, 1 or 2 secondary sensory endings were also left intact. In some experiments, the spindle was then removed to a recording chamber for further electrophysiological recording (at $22^{\circ} \mathrm{C}$; in other cases, the spindle was left in the dissecting dish, and activity was recorded continuously before, during, and after the lesioning procedure. Care was taken to ensure that no more than $4 \mathrm{hr}$ elapsed between the removal of the tenuissimus muscle and completion of the physiological recording.

The activity of each spindle was recorded for $45 \mathrm{~min}$ after the lesion, except for one spindle which was recorded for $160 \mathrm{~min}$. After physiological recording, cach spindle was immersed in a fixative consisting of $5 \%$ glutaraldehyde in $0.1 \mathrm{M}$ cacodylate buffer, with $0.1 \% \mathrm{MgCl}_{2}$. After fixing for $3 \mathrm{hr}$ at room temperature and washing overnight in buffer, the spindle was postfixed in osmium tetroxide $(0.5 \%)$ for $1.5 \mathrm{hr}$, dehydrated, and embedded in epoxy resin. Serial $4 \mu \mathrm{m}$ sections were mounted in liquid epoxy (without staining) and examined with a light microscope using phase-contrast optics. Selected sections were remounted on epoxy stubs and thin-sectioned for electron microscopy. The thin sections (silver interference color) were stained with aqueous uranyl acetate $(45 \mathrm{~min})$ and lead citrate $(4 \mathrm{~min})$. Electron microscopy was done on a Phillips 201 transmission EM, operating at $60 \mathrm{kV}$.

The recorded analog data were processed through a window discriminator (BAK Electronics) so that primary and secondary sensory units could be analyzed separately, although they had been recorded concurrently. The discriminator acceptance signal was fed into a microcomputer, in which the interspike intervals were digitized and stored. Time between spikes was measured at a resolution of $0.2 \mathrm{msec}$. Plots of instantaneous frequencies were made from the digitized data using a dot-matrix printer.

The inverse of the time between successive action potentials (instantaneous frequency) was plotted for each interspike interval. With this type of plot (Figs. 4-6), subtle changes in electrophysiological activity could be seen, but determinations of firing frequencies were somewhat subjective. For example, if instantaneous frequencies were clustered about more than one value, it was problematical to determine whether (1) the clusters were harmonic frequencies that appeared because some of the spikes in a train were aborted or not detected, or (2) there were indeed autonomous, multiplex frequencies. An oscilloscope display of the recorded analog data was useful in deciding whether harmonic clusters should have been expected, especially if 2 or more sensory units were interfering with one another. It was also helpful to plot histograms of instantaneous frequencies during a limited time period (Figs. 4, 5). 


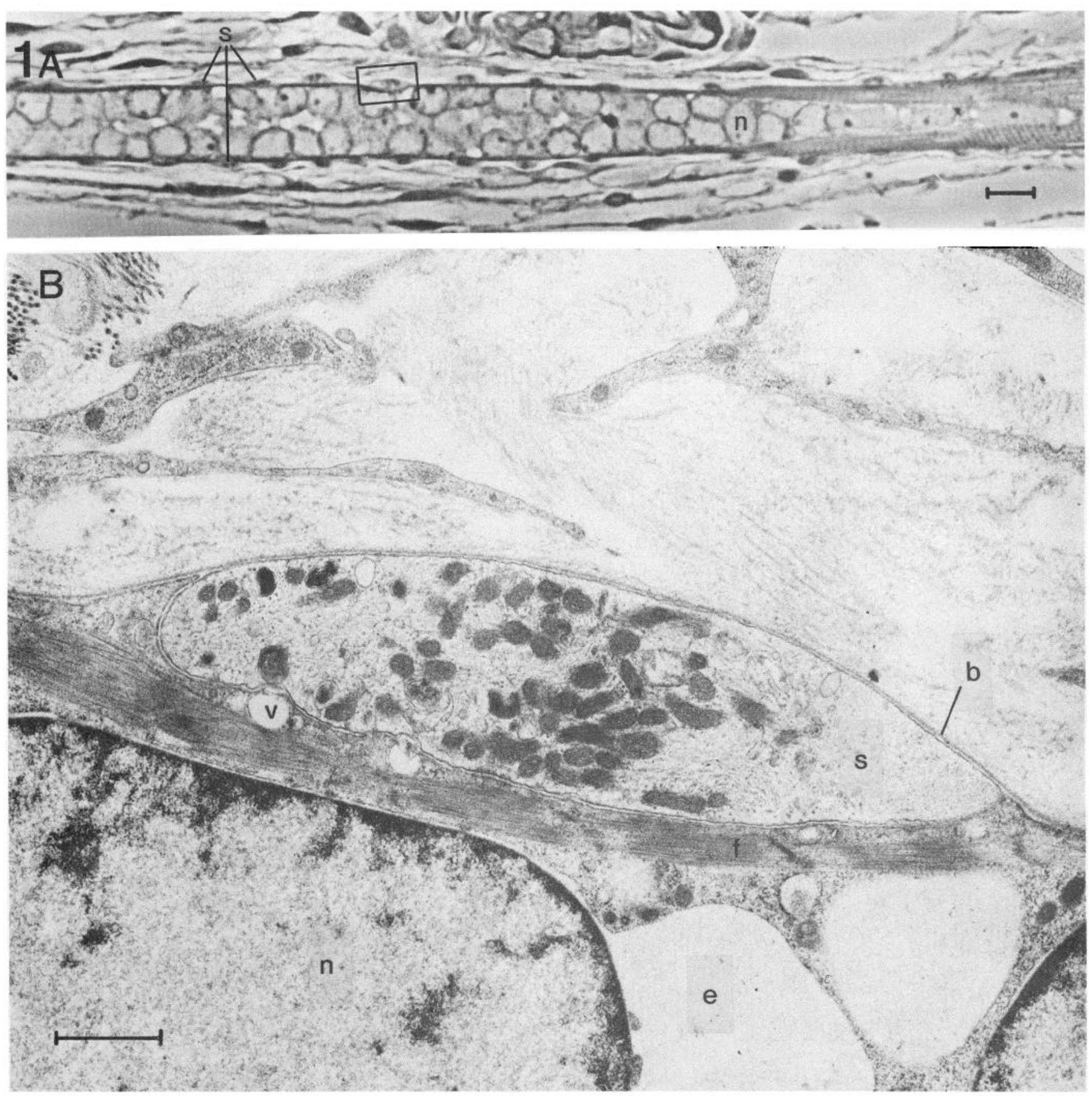

Figure 1. A, Longitudinal section, $4 \mu \mathrm{m}$, of a muscle spindle nuclear bag intrafusal muscle fiber. The spindle had been incubated in Krebs solution for $45 \mathrm{~min}$ but not lesioned. The appearance is grossly normal, except that there are clear vacuoles among the nuclei $(n)$. $s$, Sensory endings. Scale bar, $10 \mu \mathrm{m}$. $B$. Electron-micrographic enlargement of the box drawn on the light micrograph. The ultrastructure is normal, except for focal enlargements $(e)$ of the nuclear envelopes and some smaller vacuoles $(v) . n$, Nucleus; $s$, sensory ending; $f$, myofibrils; $b$, basal lamina. Scale bar, 1 $\mu \mathrm{m}$.

This revealed modal frequencies as peaks in the histogram, and the degree of scatter around the modes was made evident. If there were subharmonics of a fundamental frequency, the subharmonic modes should be expected to appear at precise fractions of the primary frequency, but the scatter, as a percentage of the model frequency, should be constant. A convenient method of quantitating scatter is to define it as a plus-or-minus percentage of modal frequency, such that the window includes $95 \%$ or more of the instantaneous frequencies assignable to that mode; such a convention has been adopted in this report.

The data presented are based on experiments with 16 spindles taken from 6 cats.

\section{Results}

\section{Light microscopy}

A control muscle spindle-i.e., one that had been dissected free and incubated in Krebs solution for $\mathbf{4 5} \mathrm{min}$ without deliberately inflicting damage-is shown in Figure $1 \mathrm{~A}$. It appears to be relatively normal by light microscopy, although some vacuoles had developed. In lesioned spindles fixed $45 \mathrm{~min}$ after transection of the intrafusal muscle fibers, the myofilaments had re- 

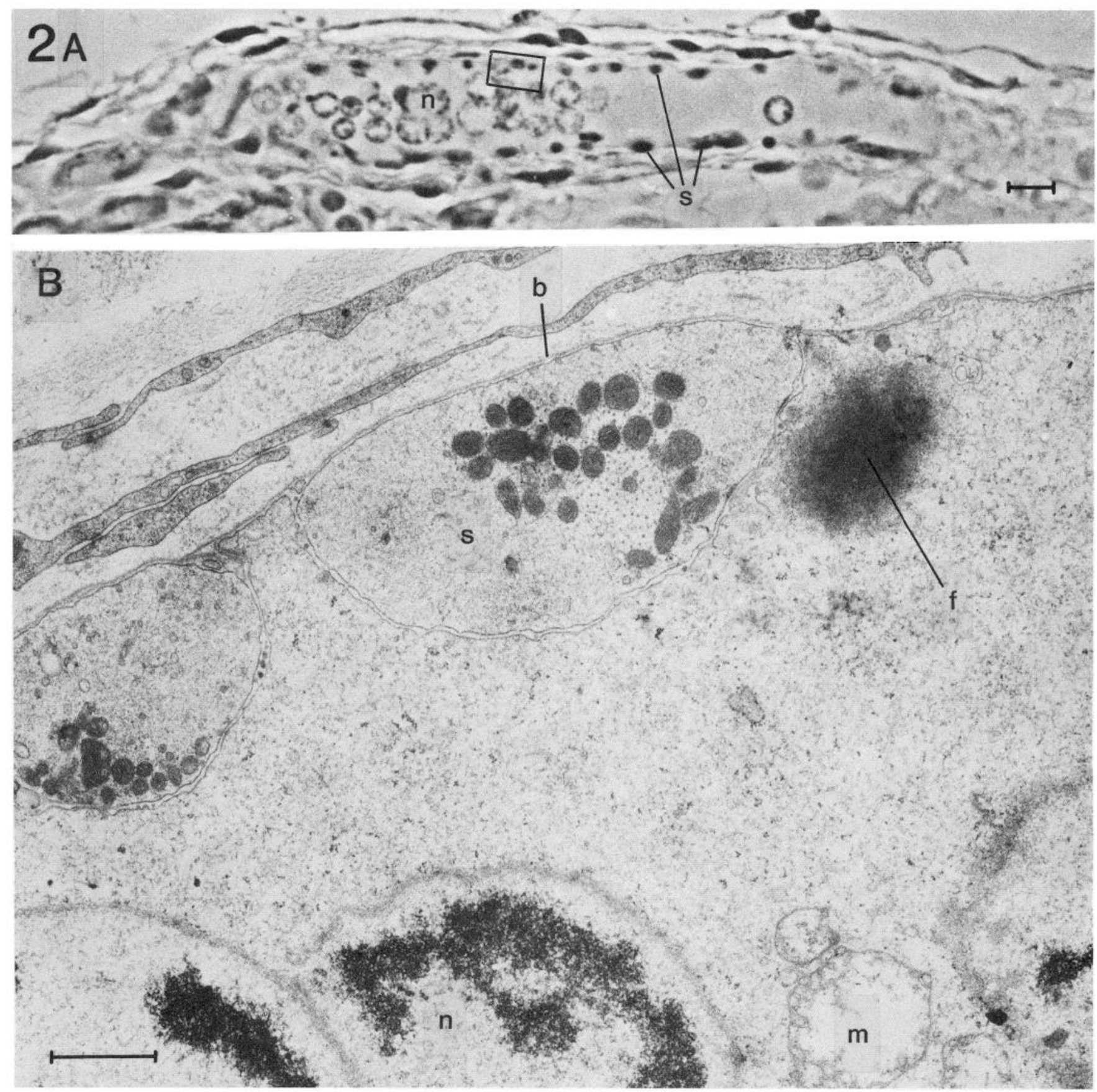

Figure 2. A, Section, $4 \mu \mathrm{m}$, of a spindle $45 \mathrm{~min}$ after lesioning the intrafusal muscle fibers. The myofibrils have been withdrawn from the sensory zone. $n$, Nuclei of a nuclear bag intrafusal fiber; $s$, sensory endings. Scale bar, $10 \mu \mathrm{m}$. $B$, Electron-micrographic enlargement of the boxed area; $m$, degenerate mitochondria; $n$, nucleus; $f$, presumed remnants of myofibrils; $s$, sensory ending; $b$, basal lamina. Scale bar, $1 \mu \mathrm{m}$.

tracted away from the sensory endings (Fig. $2 A$ ) and were aggregated in a tight mass near the lesion site. The myonuclei, which constitute "bags" and "chains" of nuclei at the equators of intrafusal muscle fibers, were somewhat dispersed but partially retained their bag and chain relationships (Fig. $2 A$ ). What was left at the equator of each muscle fiber, other than the myonuclei, was mostly a pale, homogeneous ground substance.

The sensory endings did not lose their characteristic spiral shape, and they appeared to wrap the vestiges of intrafusal muscle fibers, just as they would if the muscle fibers were intact (Fig. $2 A$ ). The spindle fixed $160 \mathrm{~min}$ after lesioning (Fig. $3 A$ ) appeared to be similar to those fixed after $45 \mathrm{~min}$.

\section{Electron microscopy}

Electron microscopy revealed that the vacuoles visible in light micrographs of control spindles (Fig. 1A) were local expansions of the nuclear envelope (Fig. 1B). Some additional, smaller vacuoles were also seen but, overall, the ultrastructural features were relatively well preserved (Fig. $1 B$ ). In contrast, the lesioned spindles were unmistakably degenerate. The ground substance that was observed in light microscopy after retraction of myofilaments was seen with electron microscopy as a finely grained flocculent material (Figs. 2B, 3B). In addition, occasional ghosts of mitochondria or other membranous organelles were seen in 

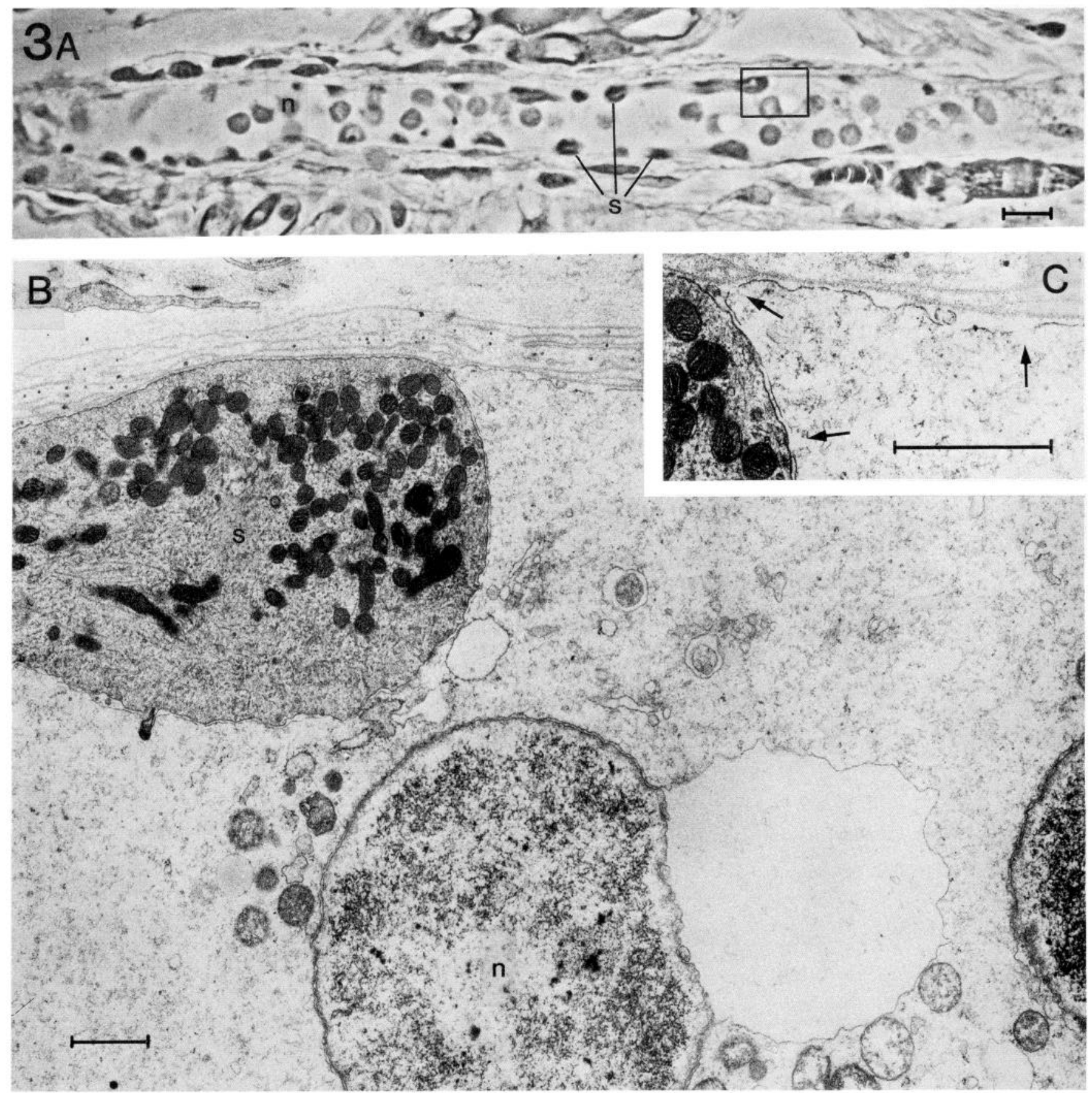

Figure 3. A, Section, $4 \mu \mathrm{m}$, of a spindle $160 \mathrm{~min}$ after lesion. There is little further degeneration compared to the $45 \mathrm{~min}$ specimens. $n$, Nuclei in a nuclear bag muscle fiber; $s$, sensory endings. Scale bar, $10 \mu \mathrm{m}$. $B$, Electron-micrographic enlargement of the box. $n$, Nucleus; $s$, sensory ending. Scale bar, $1 \mu \mathrm{m}$. $C$, Further enlargement to show breaks (arrows) in the muscle-fiber membrane. The spindle was still firing action potentials at the time of fixation.

the equatorial region (Figs. $2 B, 3 B$ ), and numerous degenerate mitochondria could be found adjacent to the remnants of the myofilament matrix, near the cut ends of intrafusal muscle fibers. The myonuclei had clumped chromatin, which was retracted from the nuclear envelope (Fig. $2 B$ ). Most of the nuclear envelopes had localized swellings (Fig. $3 B$ ). In some cases, small patches of filamentous material, presumed to be the remnants of myofilaments, were seen in the vicinity of the sensory endings; however, these masses were not observed to have any close relationship with the sensory endings.

The sensory nerve endings themselves appeared to be normal
(Figs. $2 B, 3 B$ ). The adjacent muscle fiber membrane was generally intact, although some breaks in the membrane were observed occasionally. Desmosomes could be seen to connect the sensory endings with the remnants of muscle fiber membranes (Fig. $2 B$ ). The muscle fiber membranes in some cases appeared to have become vesiculated, especially in regions adjacent to sensory endings. The basal laminae, covering both the intrafusal muscle fibers and their apposed sensory endings, appeared to be normal (Fig. $2 B$ ).

The one spindle that was incubated in Krebs solution for 160 $\min$ (Fig. $3 B$ ) showed little further degeneration, compared with 


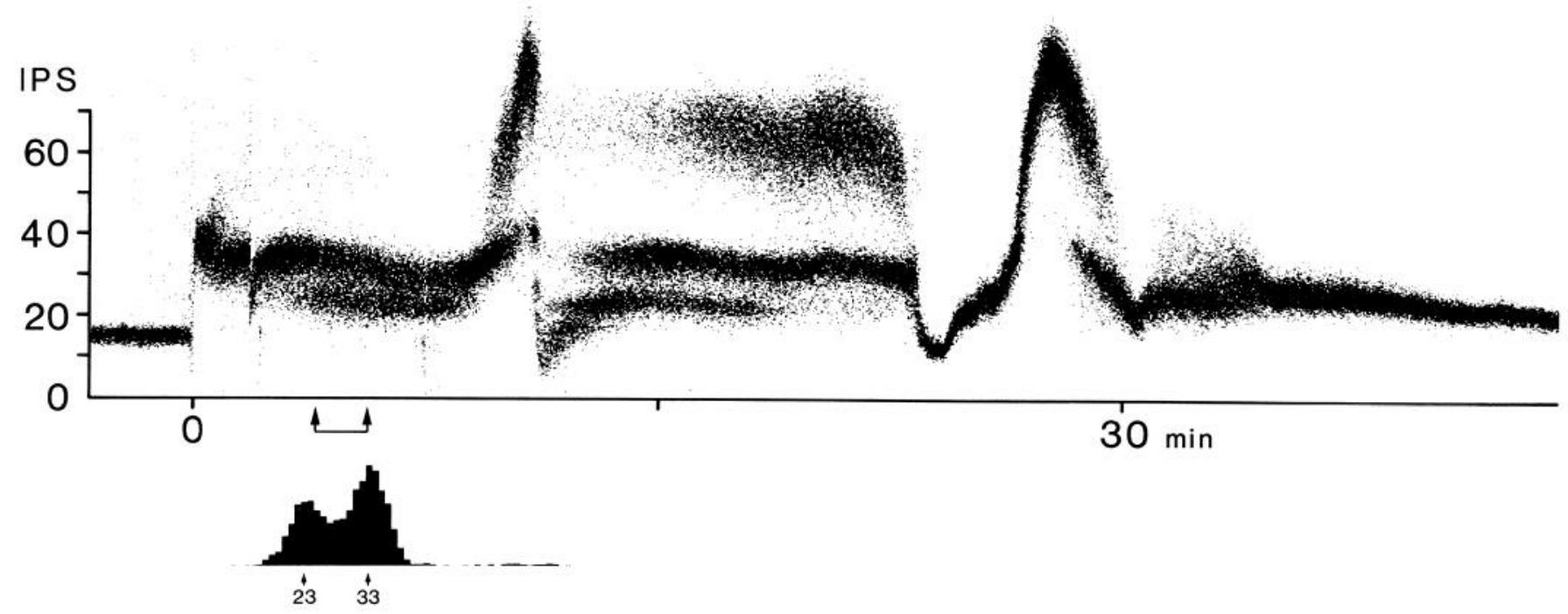

Figure 4. Instantaneous frequency-gram of the discharge from a muscle-spindle primary sensory ending. Two cuts, one on each side of the sensory zone, were made at time 0 and $2 \mathrm{~min}$ later, to effect a lesion of the intrafusal muscle fibers. The histogram is a collection of interspike intervals recorded during the indicated time period. The 2 modes are at 23 and 33 impulses/sec (IPS); they probably result from multiplexing of 2 encoders firing at these respective frequencies.

the 45 min specimens. The most noticeable changes were that the myonuclei were more euchromatic and the muscle fiber membranes had more breaks (Fig. $3 C$ ). The sensory endings still looked normal.

\section{Electrophysiology}

The sensory nerve fibers of relaxed, intact muscle spindles had a resting discharge of 7-19 impulses/sec (IPS), with scatter of $\pm 3-25 \%$. Primary sensory units were identified by virtue of being the only unit in a spindle; or, if there were more than one unit, the one with the largest spikes was assumed to be the primary and the others, secondaries. Neither primary nor secondary sensory units were consistently found to fire faster than the other, but the primaries usually fired with less regularity than secondaries, and were more likely to have a steadily declining rate of discharge. The deceleration of primary units ranged from 0 to $0.67 \mathrm{IPS} / \mathrm{min}$; secondaries declined from 0 to 0.12 IPS/min.

When spindles were transected, the immediate consequence in almost every case was a dramatic increase in firing rate of the sensory units, and an equally dramatic increase in the irregularity of instantaneous firing frequencies (Figs. 4-6). The results over the next $45 \mathrm{~min}$ were less predictable. Some units were active for a few minutes and then ceased firing, but most went through a number of episodes of changes in firing rate (Figs. 4-6). Peak firing rates during this time could be as high as 80 IPS. Often, several modal frequencies were observed for single sensory units (Figs. 4-6). In some of these cases, the modes could have resulted from subharmonic splitting of a single frequency (Fig. 6), but in others, it seems that there were multiple fundamental frequencies of firing. An example is shown in Figure 4 in which modal frequencies are evident at 23 and 33 IPS. This is not consistent with what is expected of harmonic frequencies, because the higher frequency is not a multiple of the lower, and there are no other modes that might suggest a common fundamental for the two. Furthermore, the original record showed no evidence of multiple sensory units (all spikes were of the same amplitude, there were no double-height or very closely spaced spikes), and there was not enough noise to interfere with detection of valid spikes.
Primary and secondary units in the same spindle did not necessarily fire in concert. For example, the primary might die after a few minutes, while the secondary kept firing for some time. When the primary and secondary both went through episodes of increased or decreased firing, the episodes were not precisely coincident (Figs. 5 and 6).

After $45 \mathrm{~min}$ (postlesion), the general result was that sensory units settled into a constant or slowly declining frequency of firing (Figs. 4-6). The activity during this time resembled what was observed before the lesion, in that primary units usually had more scatter than secondaries and were more likely to decelerate. However, both types of unit showed more than normal scatter during this period. In the one spindle that was recorded for $160 \mathrm{~min}$, the primary unit declined steadily after $45 \mathrm{~min}$, and then ceased spontaneous firing at $160 \mathrm{~min}$, although it would still respond to mechanical probing (Fig. 5). The secondary unit in this spindle had a nearly steady firing rate during this time and was, in fact, accelerating slightly after $160 \mathrm{~min}$ (Fig. 6).

\section{Discussion}

The principal finding reported here is that muscle spindles can produce sensory action potentials long after intrafusal muscle fibers have seriously degenerated. This confirms that the sensory endings are capable of somewhat autonomous electrophysiological activity, and the presence of a normal intrafusal muscle substrate is not essential for sensory discharge. This would seem to provide a useful model system for separating muscle- and nerve-dependent processes in the sensory activity from muscle spindles. However, it should be kept in mind that, although the intrafusal muscle fibers are grossly degenerated within $45 \mathrm{~min}$, there are still vestiges of the fibers present, including the basal lamina. In addition, the outer and inner capsules and their associated collagen and elastin fibers are preserved. These extracellular elements may have some mechanical properties that affect the functioning of sensory endings, and their possible involvement in maintenance or adaptation of firing should not be overlooked (Poppele and Quick, 1985).

A detailed characterization of the postlesion sensory discharge is not within the scope of this report. However, several interesting features were observed and deserve some comment. 

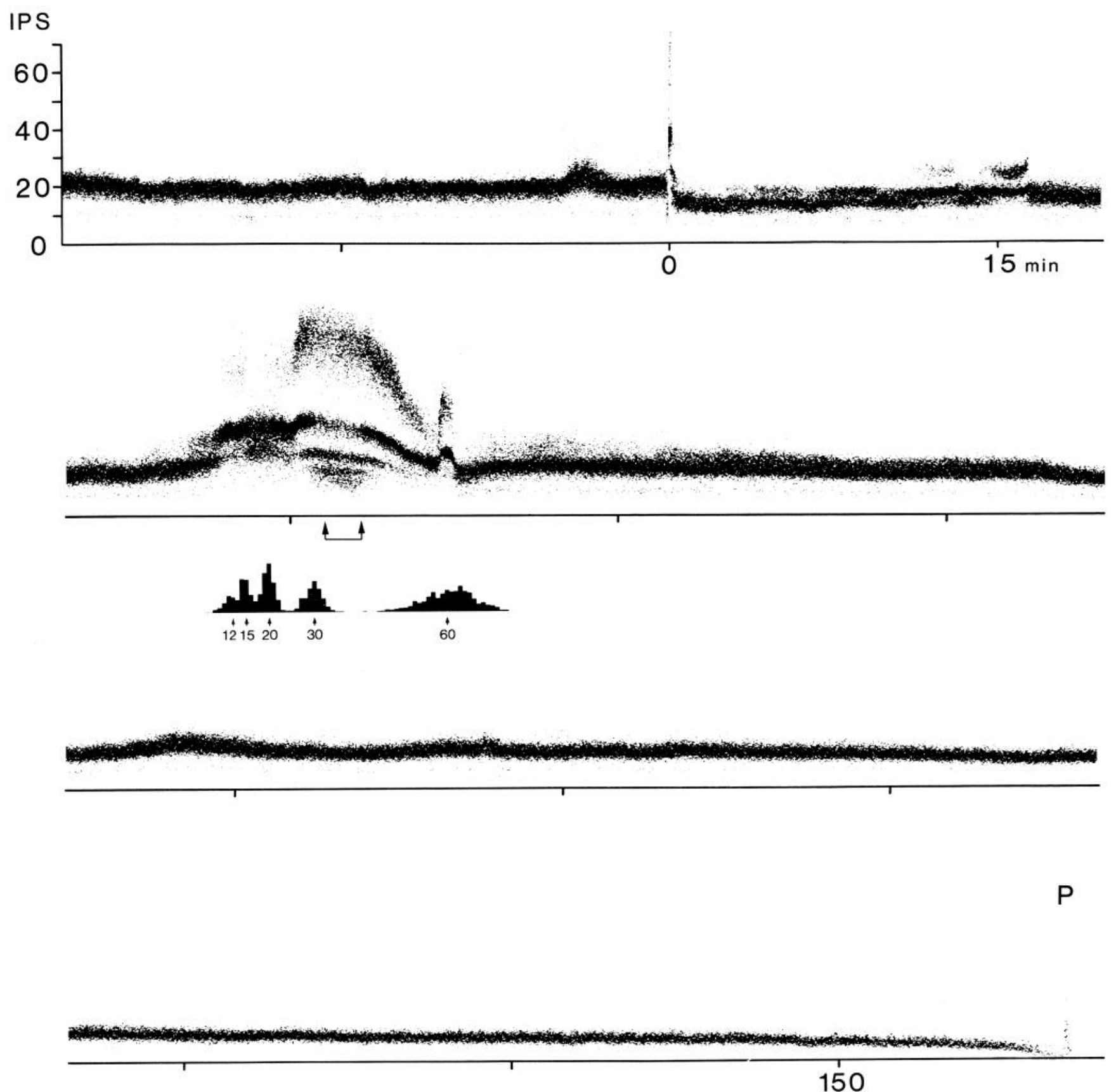

Figure 5. Frequency-gram recorded from a primary ending. Two cuts in close succession were made at time 0 to lesion the intrafusal muscle. At the end of the record, the unit stopped firing spontaneously but was still sensitive to probing (at point $P$ ). The histogram illustrates frequencies during the indicated interval. It appears that the frequencies may be harmonics of 60 impulses/sec (IPS), with modes at $60,30,20,15$, and 12 IPS.

\section{Irregular firing}

An increased variation in intervals between successive action potentials is evident following a lesion of the intrafusal muscle fibers. This may be due to any of a number of abnormal physiological processes resulting from the lesion: (1) mechanical disengagement of equatorial structures from the rest of the spindle, (2) changes in the ionic environment around the sensory endings, or (3) flow of injury currents in the lesioned muscle fibers. Following a lesion, the onset of irregularity is almost immediate, and thus it does not appear to depend on degeneration of the myofilaments. On the other hand, some degree of irregularity persists for hours, beyond the time necessary for extensive de- generation of myofibrils and the muscle fiber membrane (Figs. 2,3 ), and this persistence argues against the idea that irregularity is solely related to noisy contractions of the myofibrils or to injury currents.

\section{Episodic firing}

Usually, a number of episodes of increased firing are observed in sensory nerve fibers during a 45 -min period after lesion of the intrafusal muscle (Figs. 4-6). These episodes may be related to a degenerative process that results in withdrawal of myofilaments from the sensory zone. In some cases, it is possible to distinguish a number of discrete changes in firing rate, and when they occur, the number of such episodes is less than 10 . This 

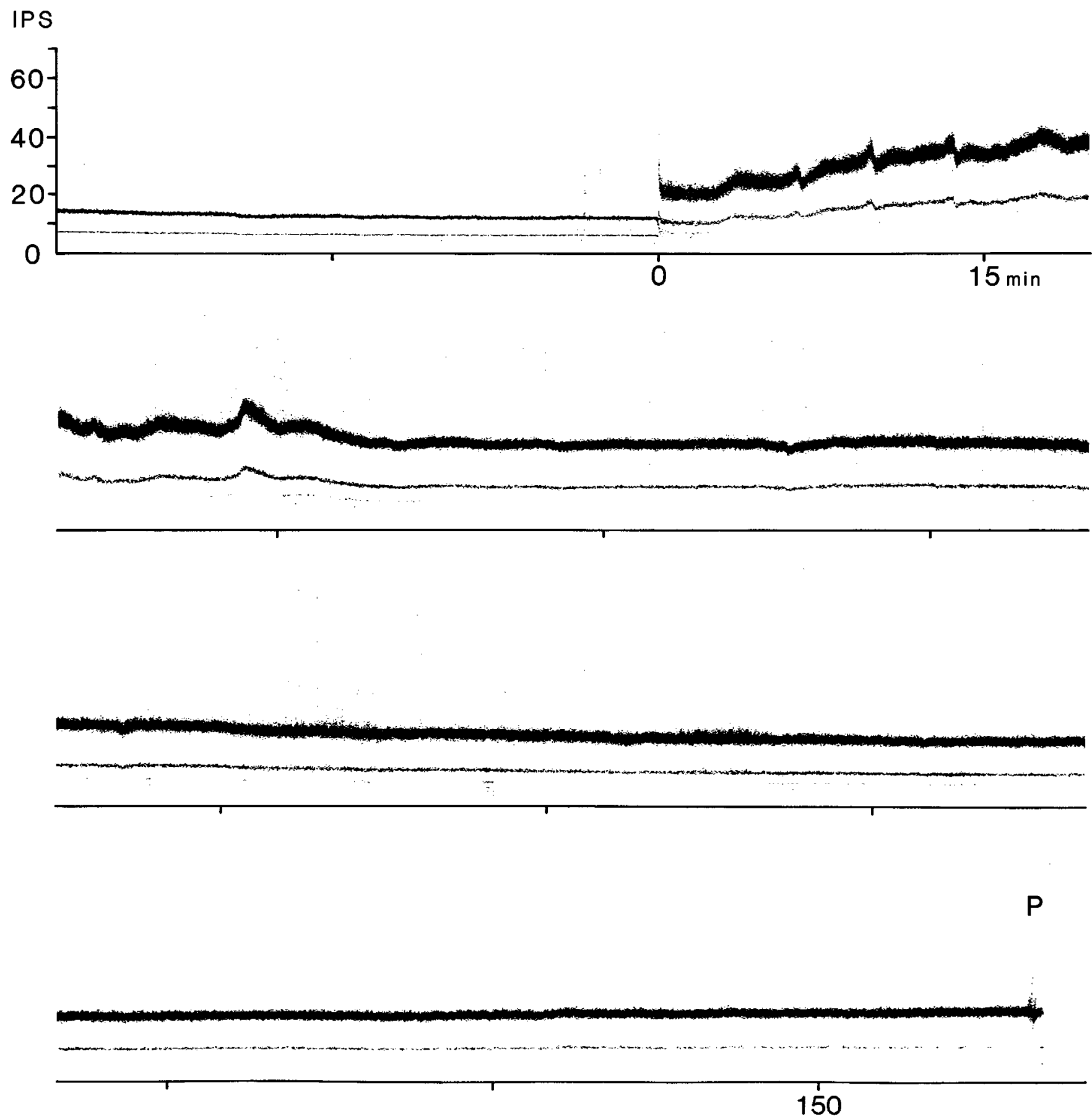

Figure 6. Frequency-gram recorded from the secondary ending of the same spindle as in Figure 5. There are prominent subharmonic instantaneous frequencies, which are artifacts of the method used to record primary and secondary units simultaneously. (Specifically, when both units fire coincidently, the single-window voltage discriminator did not recognize the event as a spike in the secondary unit.) This unit was still firing when the primary had died. It also was sensitive to probing (point $P$ ).

would be consistent with one episode corresponding to an event in a single intrafusal muscle fiber, of which there are fewer than 10.

\section{Frequency multiplexing}

The appearance of multiplex frequencies is not consistent with the usual concept of signal processing in sensory units. The units in muscle spindles are known to consist of several sensory endings, each with its own encoder site (Horak, 1977; Hulliger et al., 1977; Ito et al., 1974; Quick et al., 1980). The encoders initiate action potentials in the terminal myelinated branches of the sensory axon, and each encoder must compete with the others for access to the singular main axon projecting out of the spindle. If there were no competition, that is, if every encoder were free to send its own train of spikes into the common line, then interspike intervals in the common axon would be randomly distributed above a minimum frequency corresponding to the slowest encoder. This can be seen in a computer simulation of 2 competing encoders, one of which is set to fire at 30 IPS with $10 \%$ scatter (in a Gaussian distribution), while the 

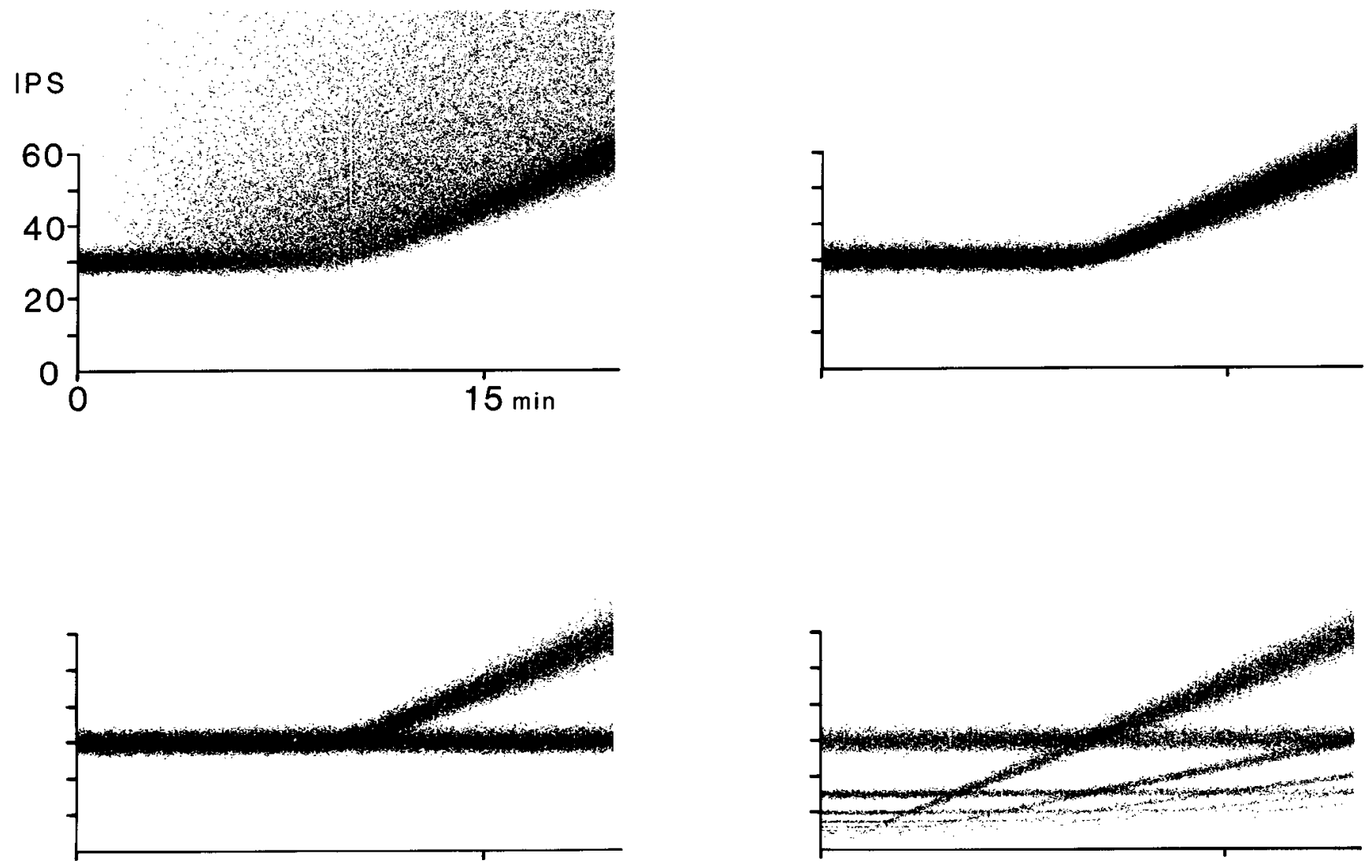

Figure 7. Computer-modeled frequency-grams of 2 competing encoders, one firing at a steady rate of $30 \mathrm{impulses} / \mathrm{sec}$ (IPS) and the other accelerating from 0 IPS at the start of the record to 60 IPS at the end. Scatter for each encoder is set at $10 \%$, in a Gaussian distribution. $A$, Neither encoder influences the other. Frequencies above 100 IPS have been filtered out. $B$, Each encoder resets the other. $C$, Each encoder resets the other, but the accelerating encoder aborts $50 \%$ of its spikes. $D$, Each encoder resets the other, and both abort $50 \%$ of their spikes.

other accelerates from 0 to 60 IPS, also with $10 \%$ scatter (Fig. $7 A$ ). Real spindles do not match that model, having no random interference of frequencies; thus, the common axon must be dominated by only one encoder at a time. This is consistent with the understanding that all spikes generated in a tree of axons should be capable of invading all of the branches (Eagles and Purple, 1974). In each case, the fastest encoder would have access to the trunk axon and would suppress access of the slower encoders by collison of spikes in their branch lines. If this is modeled by computer, again using a steady encoder competing with an accelerating encoder, it can be seen that the faster cncoder completely dominates at any particular time, and there is no high-frequency interference (Fig. $7 B$ ). In order to get multiplexing of the 2 model encoders, it is necessary to abort some of the spikes in the faster unit. If this is done, the faster encoder has some doublc-length intervals during which a slower encoder can have access to the main line. The result is an expression of both frequencies (Fig. 7C), qualitatively similar to what is seen in lesioned spindles. If both of the model encoders have occasional abortive spikes, then the frequencies of both are expressed, plus their subharmonics (Fig. 7D). This last paradigm might also occur in real lesioned spindles, although no obvious examples were observed in this study.

In the lesioned spindles, multiplexing of frequencies was quite obvious. In normal spindles, multiplexing is usually not apparent, even though it might occur to some extent. The reason why a single frequency is usually seen in normal spindles is that multiple encoders tend to fire at similar rates, and shifting dominance or mutual penetrance of encoders is masked by the background of their scatter. Nevertheless, multiplexing can occa- sionally be detected in normally firing spindles, if not by gross features of the spike train, then by averaging and filtering the recorded interspike intervals (Horak, 1977).

\section{Slow adaptation}

After the period characterized by episodic firing, the sensory units settled into a fairly stable (but still noisy) rate of firing that was nearly steady for secondary endings, but declined slowly for primaries (Figs. 5, 6). The fact that the primary and secondary units adapted differently after the muscle fibers had degenerated suggests that these adaptive properties are intrinsic to the nerve fiber, and not dependent on muscle. If both types of unit had been observed to decelerate and die at similar rates, then it might be suspected that they were subject to a general degenerative process. However, this was not the case (Figs. 5, $6)$.

\section{References}

Boyd, I. A. (1976) The mechanical properties of dynamic nuclear bag fibres, static nuclear bag fibres and nuclear chain fibres in isolated cat muscle spindles. Prog. Brain Res. 44: 33-50.

Eagles, J. P., and R. L. Purple (1974) Afferent fibers with multiple encoding sites. Brain Res. 77: 187-193.

Horak, F. M. B. (1977) Multiple encoder sites on the primary afferent of the mammalian muscle spindle. Master's thesis, University of Minnesota, Minneapolis.

Hulliger, M., P. B. C. Matthews, and J. Noth (1977) Effects of combining static and dynamic fusimotor stimulation on the response of the muscle spindle primary ending to sinusoidal stretching. J. Physiol. (Lond.) 267: 839-856.

Ito, F., N. Kanamori, and H. Kuroda (1974) Structural and functional 
asymmetries of myelinated branches in the frog muscle spindle. $J$. Physiol. (Lond.) 241: 389-405.

Poppele, R. E., and D. C. Quick (1985) Effect of intrafusal muscle mechanics on mammalian muscle spindle sensitivity. J. Neurosci. 5: 1881-1885.

Poppele, R. E., W. R. Kennedy, and D. C. Quick (1979) A determi- nation of static mechanical properties of intrafusal muscle in isolated cat muscle spindles. Neuroscience 4: 401-411.

Quick, D. C., W. R. Kennedy, and R. E. Poppele (1980) Anatomical evidence for multiple sources of action potentials in the afferent fibers of muscle spindles. Neuroscience 5: 109-115. 